\title{
On certain discontinuous wave functions
}

\author{
By A. ERdélyi.
}

\section{(Received 8th November 1946. Read 6th December 1946.)}

1. Among the many solutions of the wave equation investigated by the late Professor Bateman there is one type which has so far received little attention.

In the simplest case let $f(x, y)$ be a function defined in the whole $x, y$ plane, put $\sigma^{2}=c^{2} t^{2}-z^{2}$, and consider

$$
\begin{aligned}
W & =\frac{1}{2 \pi} \int_{0}^{2 \pi} f(x+\sigma \cos \lambda, y+\sigma \sin \lambda) d \lambda & & \text { when } \sigma^{2} \geqq 0 \\
& =0 & & \text { when } \sigma^{2}<0 .
\end{aligned}
$$

An elementary calculation shows that ${ }^{1}$

$$
f_{x x}+f_{y y}+f_{z z}-c^{-2} f_{t t}=\sigma^{-1} \frac{d}{d \lambda}\left(-f_{x} \sin \lambda+f_{y} \cos \lambda\right),
$$

and it follows that $W$ satisfies the wave equation

$$
W_{x x}+W_{y y}+W_{z z}-c^{-2} W_{t t}=0
$$

if $f$ is a continuous function of its two variables with continuous partial derivatives of the first and second orders. Bateman's contention is that $W$ satisfies the wave equation under milder restrictions on $f$.

At first (2) would seem to suggest that the continuity of, at any rate, the first order derivatives is essential for $W$ to be a wave function: such a conclusion, however, would be incorrect. If $f$, or any of its relevant partial derivatives, is discontinuous along certain curves in the $x, y$ plane, the integral must be broken up into several parts, at the points where curves of discontinuity intersect the circle with radius $\sigma$, centre $(x, y)$. These part-integrals have variable limits, and the contributions of these limits to the partial derivatives cancel the contribution arising from the differentiation of the integrand.

2. Let us consider the integral

$$
V=\int_{a}^{\beta} f(x+\sigma \cos \lambda, y+\sigma \sin \lambda) d \lambda
$$

in which $a$ and $\beta$ may depend on $x, y$, and $\sigma=\left(c^{2} t^{2}-z^{2}\right)^{\frac{1}{2}}$. We shall

\footnotetext{
1 Suffixes $x, y, z, t$ indicate partial differentiations.
} 
write $f$ briefly for $f(x+\sigma \cos \lambda, y+\sigma \sin \lambda)$ and denote partial derivatives of $f$ with respect to its two variables by $f_{1}, f_{2}, f_{11}$ etc., with the understanding that in expressions outside the integral sign the limits must be substituted for $\lambda$; we write out explicitly the contribution of the upper limit and indicate by dots that a similar contribution of the lower limit should be subtracted.

If $f$ together with its partial derivatives is continuous in the interval under consideration, we have

$$
V_{x}=\int f_{1} d \lambda+\beta_{x} f-\ldots
$$

and $V_{x x}=\int f_{11} d \lambda+\beta_{x x} f+\beta_{x}\left(2-\sigma \beta_{x} \sin \beta\right) f_{1}+\sigma \beta_{x}^{2} f_{2} \cos \beta-\ldots$

with a corresponding expression for $V_{y y}$. Also

$$
\begin{aligned}
V_{\sigma} & =\int\left(f_{1} \cos \lambda+f_{2} \sin \lambda\right) d \lambda+\beta_{\sigma} f-\ldots \\
V_{\sigma \sigma} & =\int\left(f_{11} \cos ^{2} \lambda+2 f_{12} \cos \lambda \sin \lambda+f_{22} \sin ^{2} \lambda\right) d \lambda+\beta_{\sigma \sigma} f+ \\
& +\beta_{\sigma}\left(2 \cos \beta-\sigma \beta_{\sigma} \sin \beta\right) f_{1}+\beta_{\sigma}\left(2 \sin \beta+\sigma \beta_{\sigma} \cos \beta\right) f_{2}-\ldots
\end{aligned}
$$

so that

$$
\begin{aligned}
V_{x x} & +V_{y y}+V_{z z}-c^{-2} V_{t}=V_{x x}+V_{y y}-V_{\sigma \sigma}-\sigma^{-1} V_{\sigma} \\
& =\left(\beta_{x x}+\beta_{y y}-\beta_{\sigma \sigma}-\sigma^{-1} \beta_{\sigma}\right) f+ \\
& +\left\{2\left(\beta_{x}-\beta_{\sigma} \cos \beta-\sigma^{-1} \sin \beta\right)-\sigma\left(\beta_{x}^{2}+\beta_{y}^{2}-\beta_{\sigma}^{2}-\sigma^{-2}\right) \sin \beta\right\} f_{1}+(5) \\
& +\left\{2\left(\beta_{y}-\beta_{\sigma} \sin \beta+\sigma^{-1} \cos \beta\right)+\sigma\left(\beta_{x}^{2}+\beta_{y}^{2}-\beta_{\sigma}^{2}-\sigma^{-2}\right) \cos \beta\right\} f_{2}-\ldots
\end{aligned}
$$

since the contribution of the integrals is

$$
\begin{aligned}
& \int\left\{f_{11} \sin ^{2} \lambda-2 f_{12} \cos \lambda \sin \lambda+f_{22} \cos ^{2} \lambda-\sigma^{-1}\left(f_{1} \cos \lambda+f_{2} \sin \lambda\right)\right\} d \lambda \\
& =\int \frac{d}{d \lambda}\left\{\sigma^{-1}\left(-f_{1} \sin \lambda+f_{2} \cos \lambda\right)\right\} d \lambda=\sigma^{-1}\left(-f_{1} \sin \beta+f_{2} \cos \beta\right)-\ldots
\end{aligned}
$$

3. The limits of integration are determined by the curves of discontinuities so that $\beta$ will be a root of an equation of the form

$$
h(x+\sigma \cos \beta, y+\sigma \sin \beta)=0 .
$$

Assuming that $h$ has partial derivatives of the first and second orders,

$$
\left(1-\sigma \beta_{x} \sin \beta\right) h_{1}+\sigma \beta_{x} h_{2} \cos \beta=0
$$

with two similar relations obtained by differentiation of $h=0$ with 
respect to $y$ and $\sigma$. Eliminating $h_{1}: h_{2}$ from any two of these three relations, we have

$$
\begin{aligned}
& \sin \beta-\sigma \beta_{x}+\sigma \beta_{\sigma} \cos \beta=0 \\
& \cos \beta+\sigma \beta_{y}-\sigma \beta_{\sigma} \sin \beta=0 \\
& 1-\sigma \beta_{x} \sin \beta+\sigma \beta_{y} \cos \beta=0
\end{aligned}
$$

The combination $-\sigma\left(\beta_{x}+\beta_{\sigma} \cos \beta\right)(6)+\sigma\left(\beta_{y}+\beta_{\sigma} \sin \beta\right)(7)-(8)$ results in

$$
\sigma^{2}\left(\beta_{x}^{2}+\beta_{y}^{2}-\beta_{\sigma}^{2}\right)-1=0,
$$

and the combination $-(6)_{x}-\cos \beta(6)_{\sigma}+(7)_{y}+\sin \beta(7)_{\sigma}+\beta_{\sigma}(8)$ in

$$
\sigma\left(\beta_{x x}+\beta_{y y}-\beta_{\sigma \sigma}\right)-\beta_{\sigma}=0
$$

and the right-hand side of (5) is seen to vanish identically.

Thus we have proved that $W$, being a sum of integrals of the form (4), is certainly a solution of the wave equation if $f$ is continuous and possesses continuous first and second order partial derivatives except at a finite number of "smooth" curves, i.e. curves with a continuously turning tangent. Even an infinity of such curves is admissible provided that they are placed so that any circle cuts only a finite number of them. For $t=0, W$ vanishes outside the plane $z=0$ and is equal to $f$ in that plane; $W_{t}=0$ everywhere at $t=0$.

4. Bateman's more interesting results refer to the corresponding problem for a spherical surface rather than a plane.

Let $f$ be a function defined on the sphere $S$ with radius $a$, centre at the origin, $P$ any point at distance $r$ from the origin, $C$ the locus of all points on $S$ whose distance from $P$ is $c t, \bar{f}$ the mean value of $f$ over $C$, and

$$
W=\frac{a}{r} \tilde{f} \quad \text { if }|a-c t| \leqq r \leqq a+c t, \quad \text { and }=0 \text { otherwise. }
$$

Of this function Bateman says " in all cases that have been examined $W$ has been found to be a solution of the wave equation ... and to satisfy the initial conditions (for $t=0$ ) $W=f$ when $P$ is on $S, W=0$ when $P$ is not on $S, W_{t}=0$ everywhere."

From Bateman's work it follows that if $(r, \theta, \phi)$ are spherical

1. H. Bateman, Ann. of Maths. (2) 31, 158.162 (1930) (where the factor $a$ is omitted), and Partial Differential Equations of Mathematical Physics (1932) p. 189 Examples. 
polar coordinates, $\Sigma Y_{n}(\theta, \phi)$ the expansion of $f$ in spherical surface harmonics, and $\cos \gamma=\left(a^{2}+r^{2}-c^{2} l^{2}\right) /(2 a r)$, then

$$
W=\frac{a}{r} \Sigma P_{n}(\cos \gamma) Y_{n}(\theta, \phi) \quad(|a-c t| \leqq r \leqq a+c t)
$$

and hence that $W$ satisfies the wave equation provided that it is permissible to perform the partial differentiations term-by-term.

An alternative representation of $W$ is as follows. Let $f$ be given as a function of the stereographic coordinates $\xi=\tan \frac{1}{2} \theta \cos \phi$, $\eta=\tan \frac{1}{2} \theta \sin \phi$, so that $f=f(\xi, \eta)$. Then

$$
W=\frac{a}{2 \pi r} \int_{0}^{2 \pi} f(X, Y)-\frac{(\cos \theta+\cos \gamma) d \lambda}{1+\cos \theta \cos \gamma+\sin \theta \sin \gamma \cos (\phi-\lambda)}
$$

with $X=\frac{\sin \theta \cos \phi+\sin \gamma \cos \lambda}{\cos \theta+\cos \gamma}, Y=\frac{\sin \theta \sin \phi+\sin \gamma \sin \lambda}{\cos \theta+\cos \gamma}$

for $|a-c t| \leqq r \leqq a+c t$, and this integral may serve to prove Bateman's result for functions $f$ which have discontinuities along certain curves on $S$. That $W$ remains a wave function for such functions, Bateman conjectured and made plausible by considering special examples.

Mathematical Institute,

The University, Edinburgh, 1. 